Vietnam Journal of Mechanics, VAST, Vol.39, No.3 (2017), pp. $203-214$

DOI:10.15625/0866-7136/8422

\title{
NUMERICAL AND EXPERIMENTAL STUDIES FOR CRACK DETECTION OF A BEAM-LIKE STRUCTURE USING ELEMENT STIFFNESS INDEX DISTRIBUTION METHOD
}

\author{
Nguyen Viet Khoa*, Nguyen Van Quang, Nguyen Dinh Kien, \\ Cao Van Mai, Dao Thi Bich Thao \\ Institute of Mechanics, Vietnam Academy of Science and Technology, Hanoi, Vietnam \\ *E-mail: nvkhoa@imech.ac.vn \\ Received June 21, 2016
}

\begin{abstract}
In this paper, numerical and experimental studies for crack detection of structures using "element stiffness index distribution" are presented. The element stiffness index distribution is defined as a vector of norms of sub-matrices corresponding to element stiffness matrices calculated from the reconstructed global stiffness matrix of the beam. When there is a crack at an element, the element stiffness index of that element will be changed. By inspecting the change in the element stiffness index distribution, the crack can be detected. A significant peak in the element stiffness index distribution is the indicator of the crack existence. The crack location is determined by the location of the peak and the crack depth can be determined from the height of the peak. The global stiffness matrix is calculated from the measured frequency response functions instead of mode shapes to avoid limitations of the mode shape-based methods for crack detection. Numerical simulation results for the cases of beam-like structures are provided. The experiment is carried out to justify the efficiency of the proposed method.
\end{abstract}

Keywords: Crack detection, damage detection, multi-cracks detection, stiffness method, element stiffness, element stiffness index distribution.

\section{INTRODUCTION}

The subject of structural crack detection has attracted many researches in the last three decades [1]. When there are cracks, the dynamic properties such as frequency and mode shape are changed. The changes in dynamic properties of structures are very useful for crack detection [2]. Many of the studies for crack detection were concentrated on the analysis of natural frequency in the case of open crack for simplification reasons. Liang et al. [3] proposed theoretical relationships between eigenfrequency changes and magnitudes, and the locations of crack-induced damage for beam structures with different boundary conditions. $\mathrm{Hu}$ and Liang [4] presented an integrated method using the change in natural frequency for crack detection. In this work, the spring model and

(C) 2017 Vietnam Academy of Science and Technology 
continuum damage model were combined to analyze crack positions and crack depths. Ruotolo and Surace [5] proposed a multi-crack detection method using finite element model of the structure and genetic algorithms. A crack detection approach based on the minimization of the difference between measured data and a computational model of a beam-type structure was proposed by Shen and Taylor [6]. Some authors $[7,8]$ modeled cracks as massless rotational springs, whose stiffness were calculated using fracture mechanics to study the natural frequencies and mode shapes of cracked beams.

Although the natural frequency can be obtained easily for detecting the existence of cracks, however, it is difficult for determining the location of cracks. Meanwhile, the mode shape is distorted locally at the crack location and it can be used for localizing the crack. Some authors applied successfully mode shapes for crack detection. Kisa et al. [9] presented a method for free vibration analysis of cracked beams using a combination of finite elements and component mode synthesis methods. Pandey et al. [10] presented the damage detection method using mode shape curvature. The reduction in cross section caused by the damage leads to an increase in the curvature of the mode shapes in the vicinity of the damage. In other papers [11,12], the change in curvature of mode shapes was used to determine the location of the crack. Some other used stiffness and flexibility matrices of structures obtained from mode shapes for crack detection purpose since these matrices are related directly to cracks in structures. Pandey and Biswas [13] presented a method for crack detection based on the difference between the flexibility matrices of the undamaged and damaged structures. Jaishi and Ren [14] used modal flexibility residual for damage detection. Recently, Caddemi and Caliò [15] proposed the method to localize the exact multiple cracks using frequency and mode shape measurements. However, the mode shape-based methods for crack detection have some limitations. The local change in a mode shape caused by a crack is very small and it can only be inspected visually with a very large crack depth $[8,16]$. There will be an error in the stiffness and flexibility matrices calculated from mode shapes since only some fundamental modes can be measured with a high accuracy in practice.

To overcome this issue, a method for crack detection using "stiffness index distribution" which is obtained from the measured frequency response functions, not from the mode shapes as the previous methods to avoid the above limitations of the mode shapes, has been proposed by the authors of this paper [17]. The numerical simulation results obtained by the proposed method were promising. However, no validation by the ex-

periment has been carried out and the applied structures in this case studies were very simple. In this paper, the numerical study for a more complex structure using the "stiffness index distribution" method is presented. The experiment is carried out to justify the proposed method. Good agreement is obtained between numerical and experimental results.

\section{ELEMENT STIFFNESS INDEX DISTRIBUTION}

In finite element model the governing equation of a beam-like structure can be written as follows

$$
\mathbf{M} \ddot{\mathbf{y}}(t)+\mathbf{C} \dot{\mathbf{y}}(t)+\mathbf{K y}(t)=\tilde{\mathbf{f}}(t),
$$


where $\tilde{\mathbf{f}}=\sum_{e} \mathbf{T}_{e}^{T} \mathbf{f} ; \mathbf{f}=\int_{L_{e}} N_{e}^{T} f_{e} d x ; \mathbf{M}, \mathbf{C}$ and $\mathbf{K}$ are structural mass, damping and stiffness matrices; $f$ is the excitation force; $N_{e}^{T}$ is the transposition of the shape functions at the position $x$ of the interaction force; $y$ is the nodal displacement of the beam.

The concept of "element stiffness index distribution" is briefly presented here, more details can be found in the previous work [17]. If the $i^{\text {th }}$ element stiffness matrix is denoted as

$$
\mathbf{K}_{e}^{i}=\left[\begin{array}{llll}
k_{11}^{i} & k_{12}^{i} & k_{13}^{i} & k_{14}^{i} \\
k_{21}^{i} & k_{22}^{i} & k_{23}^{i} & k_{24}^{i} \\
k_{31}^{i} & k_{32}^{i} & k_{33}^{i} & k_{34}^{i} \\
k_{41}^{i} & k_{42}^{i} & k_{43}^{i} & k_{44}^{i}
\end{array}\right]
$$

then any sub-matrix in the global stiffness matrix $\mathbf{K}$ can be expressed as follows

$$
\tilde{\mathbf{K}}_{e}^{i}=\left[\begin{array}{cccc}
\left(k_{33}^{i-1}+k_{11}^{i}\right) & \left(k_{34}^{i-1}+k_{12}^{i}\right) & k_{13}^{i} & k_{14}^{i} \\
\left(k_{43}^{i-1}+k_{21}^{i}\right) & \left(k_{44}^{i-1}+k_{22}^{i}\right) & k_{23}^{i} & k_{24}^{i} \\
k_{31}^{i} & k_{32}^{i} & \left(k_{33}^{i}+k_{11}^{i+1}\right) & \left(k_{34}^{i}+k_{12}^{i+1}\right) \\
k_{41}^{i} & k_{42}^{i} & \left(k_{43}^{i}+k_{21}^{i+1}\right) & \left(k_{44}^{i}+k_{22}^{i+1}\right)
\end{array}\right] .
$$

This sub-matrix is constructed mainly from the $i^{\text {th }}$ element stiffness matrix with some additional components of the $(i-1)^{t h}$ and $(i+1)^{\text {th }}$ element stiffness matrices. Since the sub-matrix $\tilde{\mathbf{K}}_{e}^{i}$ reflects the local stiffness, its norm should serve as a good local indication of its stiffness condition. Therefore, the change in norm of the sub-matrix $\tilde{\mathbf{K}}_{e}^{i}$ can be related to the change in the element stiffness, or in other words, it can be related to the damage at the $i^{t h}$ element. In order to detect the change in norm of the sub-matrix $\tilde{\mathbf{K}}_{e}^{i}$ from the global stiffness matrix, we define an element stiffness index distribution as follows

$$
\Lambda=\left[\begin{array}{llll}
\lambda_{1} & \lambda_{2} & \ldots & \lambda_{Q}
\end{array}\right]
$$

where $\lambda_{i}=\operatorname{norm}\left(\tilde{\mathbf{K}}_{e}^{i}\right)$ is called the $i^{\text {th }}$ element stiffness index; $Q$ is the number of finite elements. When there is no crack the element $\lambda_{i}$ is the same for every intact element. However, when there is a crack at the $i^{\text {th }}$ element, $\lambda_{i}$ will be changed while the other submatrices remain the same. Therefore, the element stiffness index distribution is expected to have a significant change at the $i^{\text {th }}$ element.

The global stiffness matrix can be reconstructed from Eq. (1) in the frequency domain as follows

$$
\mathbf{K H}(\omega)=\mathbf{L}+\omega^{2} \mathbf{M H}(\omega)-i \omega \mathbf{C H}(\omega),
$$

where $\mathbf{H}(\omega)=\left[\begin{array}{llll}H_{1} & H_{2} & \ldots & H_{n}\end{array}\right]^{T}$ is a vector of measured frequency response functions at frequency $\omega$ obtained by acting as an external force at the fixed $k^{\text {th }}$ degree of freedom with the response signals being measured at all of the degrees of freedom. $\mathbf{L}$ is a constant vector with the $k^{\text {th }}$ component equal to unity and the other components equal to zero. It is assumed that the crack only influences the stiffness matrix but it does not influence the 
mass and damping matrices. Thus, we can consider that $\mathbf{H}, \mathbf{M}$ and $\mathbf{C}$ are known, then the stiffness matrix K can be calculated step by step from (5) as follows.

In the finite element method, the matrix $\mathbf{K}$ has only four non-zero components in each row of the first and the last two rows. While, there are six non-zero components in the middle of $\mathbf{K}$. The components in the first row of $\mathbf{K}$ are obtained from the following equation

$$
\hat{\mathbf{K}}_{1}=\mathbf{A}_{1}^{-1} \mathbf{B}_{1},
$$

where

$$
\begin{aligned}
& \hat{\mathbf{K}}_{1}=\left(k_{11} k_{12} k_{13} k_{14}\right)^{T} \text {, } \\
& \mathbf{A}_{1}=\left[\begin{array}{llll}
H_{1}\left(\omega_{1}\right) & H_{2}\left(\omega_{1}\right) & H_{3}\left(\omega_{1}\right) & H_{4}\left(\omega_{1}\right) \\
H_{1}\left(\omega_{2}\right) & H_{2}\left(\omega_{2}\right) & H_{3}\left(\omega_{2}\right) & H_{4}\left(\omega_{2}\right) \\
H_{1}\left(\omega_{3}\right) & H_{2}\left(\omega_{3}\right) & H_{3}\left(\omega_{3}\right) & H_{4}\left(\omega_{3}\right) \\
H_{1}\left(\omega_{4}\right) & H_{2}\left(\omega_{4}\right) & H_{3}\left(\omega_{4}\right) & H_{4}\left(\omega_{4}\right)
\end{array}\right], \\
& \mathbf{B}_{1}=\left[\begin{array}{c}
L_{1}\left(\omega_{1}\right)+\sum_{j=1}^{4} \omega_{1}^{2} m_{1 j} H_{j}\left(\omega_{1}\right)-\sum_{j=1}^{4} i \omega_{1} c_{1 j} H_{j}\left(\omega_{1}\right) \\
L_{1}\left(\omega_{2}\right)+\sum_{j=1}^{4} \omega_{2}^{2} m_{1 j} H_{j}\left(\omega_{2}\right)-\sum_{j=1}^{4} i \omega_{2} c_{1 j} H_{j}\left(\omega_{2}\right) \\
L_{1}\left(\omega_{3}\right)+\sum_{j=1}^{4} \omega_{3}^{2} m_{1 j} H_{j}\left(\omega_{3}\right)-\sum_{j=1}^{4} i \omega_{3} c_{1 j} H_{j}\left(\omega_{3}\right) \\
L_{1}\left(\omega_{4}\right)+\sum_{j=1}^{4} \omega_{4}^{2} m_{1 j} H_{j}\left(\omega_{4}\right)-\sum_{j=1}^{4} i \omega_{4} c_{1 j} H_{j}\left(\omega_{4}\right)
\end{array}\right],
\end{aligned}
$$

and $k_{i j}, m_{i j}, c_{i j}, L_{i}\left(\omega_{j}\right), H_{i}\left(\omega_{j}\right)$ are the components of $\mathbf{K}, \mathbf{M}, \mathbf{C}, \mathbf{L}$ and $\mathbf{H}$, respectively. Similar procedure is applied for the next rows of $\mathbf{K}$.

\section{REGULARIZATION}

When the measured signals are contaminated by noise, both $\mathbf{A}$ and $\mathbf{B}$ in Eq. (6) will contain errors and these equations subsequently become ill-conditioned problem. Therefore, we need to apply regularization methods which are available in published literature. One of the most popular methods is Tikhonov regularization [18].

It is noted that the noise in Eq. (6) comes mainly from the frequency response function $\mathbf{H}$. Therefore, it is important to filter out the noise of $\mathbf{H}$ before applying the regularization. In this scenario the truncated singularity value decomposition method (TSVD) can be applied to reduce the noise in $\mathbf{H}$. The matrices $\mathbf{U}, \mathbf{S}, \mathbf{V}$ are first decomposed from matrix $\mathbf{H}$ so that

$$
\mathbf{H}=\mathbf{U S V}^{T} \text {. }
$$

In discrete ill-posed problems, the singular values of the matrix $\mathbf{H}$ decay gradually to zero. The largest singularity values correspond to the smooth components of $\mathbf{H}$ while the smaller values correspond to the noise in $\mathbf{H}$. Therefore, in order to filter out the noise the tiny positive singular values are set to zero [19]

$$
\sigma_{i}= \begin{cases}\sigma_{i}, & i \leq \lambda \\ 0, & \text { otherwise }\end{cases}
$$


where $\sigma_{i}$ is the singularity of matrix $\mathbf{S}$. $\lambda$ is chosen so that $\sigma_{\lambda} \gg \sigma_{\lambda+1}$. The matrix $\mathbf{H}$ is then reconstructed from (8).

After filtering out the noise from $\mathbf{H}$, the Tikhonov regularization method is applied to solve Eq. (6). Tikhonov regularization method used to solve the problem is denoted as

$$
\min _{x \in \mathbb{R}^{n}}\left\{\|\mathbf{A x}-\mathbf{B}\|^{2}+\left\|\mathbf{L}_{\mu} \mathbf{x}\right\|^{2}\right\}
$$

where $L_{\mu} \in \mathbb{R}^{m \times n}, m \leq n$, is called the regularization matrix. In this study $m$ and $n$ are the dimensions of $\mathbf{A}$ in Eq. (10) and $m=n$. The scalar $\mu>0$ is known as the regularization parameter. The standard form of Tikhonov regularization takes $\mathbf{L}_{\mu}=\mu \mathbf{I}$, where $\mathbf{I}$ is the unit matrix. When singularity value decomposition (SVD) of $\mathbf{A}$ is available, i.e. $\mathbf{A}=$ $\mathbf{U S V}^{T}$, the explicit solution of (10) is given by

$$
\mathbf{x}=\mathbf{V}\left(\mathbf{S}^{T} \mathbf{S}+\mu^{2} \mathbf{I}\right)^{-1} \mathbf{S}^{T} \mathbf{U}^{T} \mathbf{B} .
$$

In order to find $\mu$, the norm $\|e\|=\varepsilon$ of the error in $\mathbf{B}$ is first estimated from the simulation solution $\mathbf{x}_{\mu}$ and the measurement $\mathbf{x}$, then $\mu$ will be chosen so that

$$
\left\|\mathbf{A} x_{\mu}-\mathbf{B}\right\| \leq \eta \varepsilon
$$

where $\eta>1$ is user-specified constant which is independent of $\varepsilon . \eta=1$ means to find the exact parameter $\mu$ which may not always happen in practice since the measurement noise is random. Meanwhile, $\eta \gg 1$ will lead to the inaccuracy of parameter $\mu$. Therefore, $\eta$ will be chosen based on a particular problem. Finally, parameter $\mu$ is calculated from the following equation

$$
\sum_{j=1}^{n}\left(\frac{\sigma_{j}^{2}}{\sigma_{j}^{2}+\mu^{2}}-1\right)^{2} b_{j}^{2}=\eta^{2} \varepsilon^{2},
$$

\section{NUMERICAL SIMULATION}

A numerical example of a slender symmetric structure acted at the middle point by the force in the X-direction as presented in Fig. 1 is investigated. The frequency response functions in the $X$-direction are obtained along the structure. This structure consists of four columns and braced elements with the dimensions of $0.25 \mathrm{~m} \times 0.25 \mathrm{~m} \times 3.6 \mathrm{~m}$. The columns and braced elements of this structure are modelled as frame elements in the finite element analysis. The cross section of column and braced elements are $0.02 \mathrm{~m} \times$ $0.02 \mathrm{~m}$ and $0.02 \mathrm{~m} \times 0.002 \mathrm{~m}$, respectively. The material properties of the elements are: mass density $\rho=7855 \mathrm{~kg} / \mathrm{m}^{3}$; modulus of elasticity $E=2.1 \times 10^{11} \mathrm{~N} / \mathrm{m}^{2}$. The number of elements used in this simulation is 240 . Each column is divided by 36 elements. It is assumed that there is a crack located at the $17^{\text {th }}$ element in column \#1. Here, the elements in column \#1 are numbered ascendantly from the bottom to the top of the structure. The stiffness matrix of the cracked element is adopted from [20]. Five levels of the crack depth ranging from $10 \%$ to $50 \%$ are investigated. These five cases are numbered as in Tab. 1. With this configuration of the force and the measurement points, the slender symmetric structure can be considered equivalently as a cantilever beam-like structure and the proposed method is then applied for crack detection. 


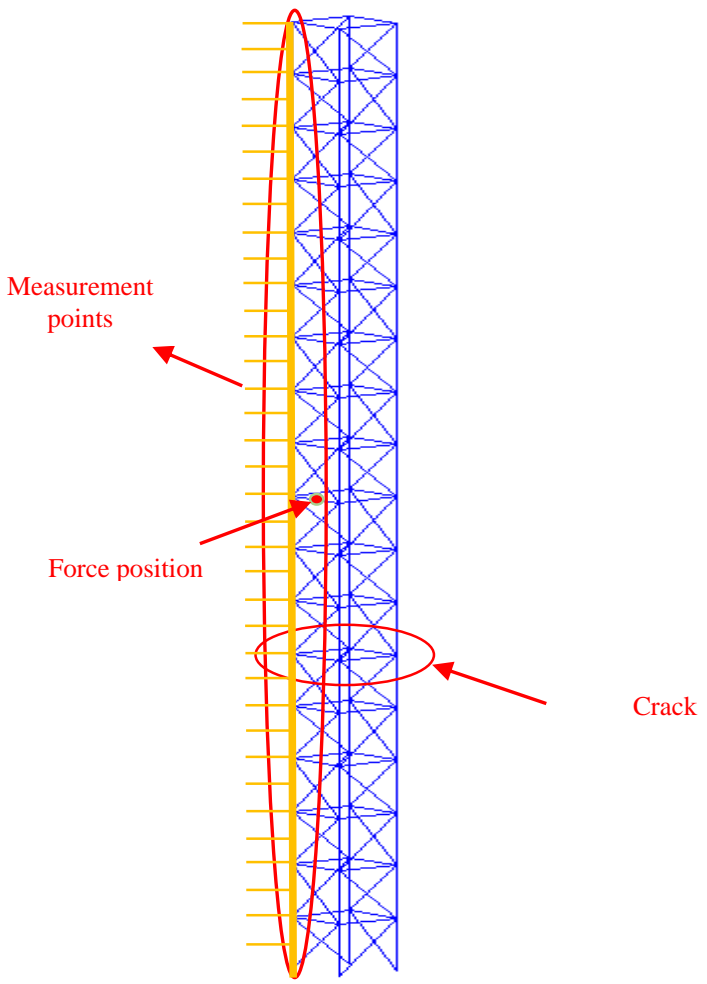

Fig. 1. The high slender structure model

Table 1. Five cases with cracks of varying depths at the crack position

\begin{tabular}{|c|c|}
\hline Case & Crack depth (\%) \\
\hline 1 & 10 \\
2 & 20 \\
3 & 30 \\
4 & 40 \\
5 & 50 \\
\hline
\end{tabular}

In this case study, the stiffness matrix $\mathbf{K}$ is reconstructed from the finite element matrices $\mathbf{M}, \mathbf{C}$ and simulation frequency response functions $\mathbf{H}$ obtained from 36 measurement points along column \#1. The element stiffness index distribution is then calculated from Eqs. (4) and (6). Fig. 2 presents the normalized element stiffness index distribution calculated from the reconstructed matrix $\mathbf{K}$ with five levels of the crack depth. As can be seen from this figure, there is a clear peak in the element stiffness index distribution at the $17^{\text {th }}$ element for every level of the crack. It can also be observed from Fig. 2 that, when the crack depth increases, the peak in the element stiffness index distribution becomes sharper. One can define the height of peak $d h$ as the difference between the values 


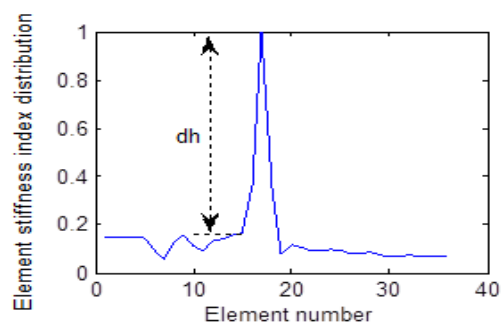

(a)

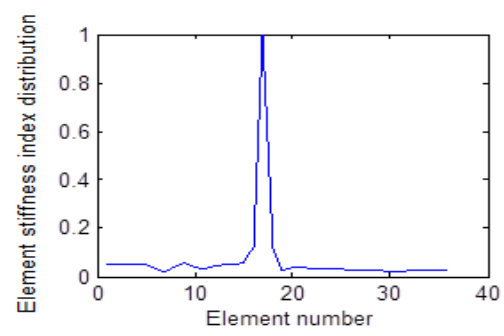

(c)

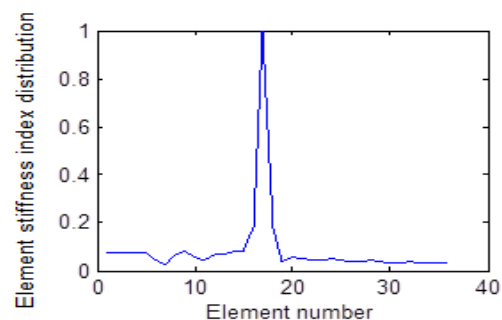

(b)

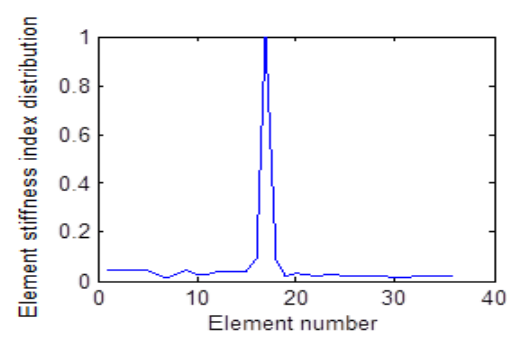

(d)

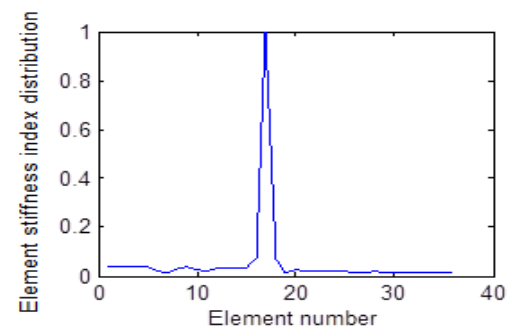

(e)

Fig. 2. Reconstructed element stiffness index distribution, element \#17 is cracked: a) crack depth $10 \%$; b) crack depth $20 \%$; c) crack depth $30 \%$; d) crack depth $40 \%$; e) crack depth $50 \%$

at the top and the bottom of the peak. Clearly, when the crack depth increases, the height of the peak increases. Therefore, the height of the peak in the element stiffness index distribution can be considered as an intensity factor which relates the change in the element stiffness index distribution at the cracked element to the crack depth. Establishing a graph of the height of the peak versus crack depth from Fig. 2, a relationship between the height of the peak (or intensity factor) and crack depth is obtained as shown in Fig. 3. It can be seen that this relationship is a straight line. This relationship is useful for crack depth estimation.

It is noted that the accuracy of the detected crack position depends on the number of measurement points since only the cracked element is determined, not the crack position. However, the accuracy of the crack position can be improved by repeating the proposed method on the crack area with more measurement points. Once the cracked element has been detected, the proposed method can be applied again to only the cracked 


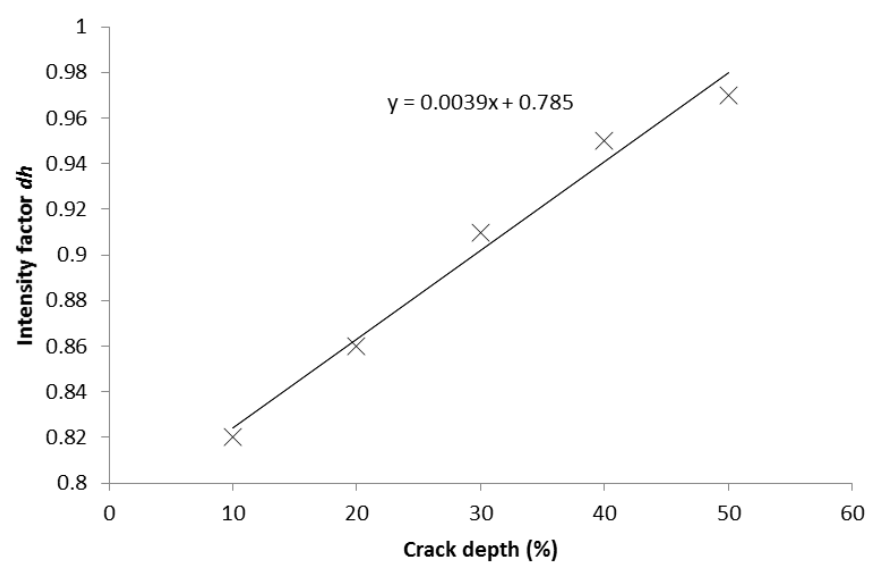

Fig. 3. The height of the first peak versus crack depth

element to determine the more accurate position of crack. This implies that, in practice the proposed method can be used as a first step to detect the crack existence and the crack area. The more intensive investigation on the crack area can be made by repeating the proposed method with more measurement points to determine the more accurate position of crack.

\section{EXPERIMENTAL VALIDATION}

In order to justify the application of the proposed method in practice, a symmetric steel structure was made in the laboratory of the Institute of Mechanics, Vietnam Academy of Science and Technology as seen in Fig. 4. The global dimensions of the structure are $0.25 \mathrm{~m} \times 0.25 \mathrm{~m} \times 3.6 \mathrm{~m}$. This structure consists of 4 columns connected rigidly together by 144 braced elements. The cross section of column and braced elements are $0.02 \mathrm{~m} \times 0.02 \mathrm{~m}$ and $0.02 \mathrm{~m} \times 0.002 \mathrm{~m}$, respectively. The vibration instrument system is incorporated into the experimental set up as presented in Fig. 5. The vibration instrument system consists of the Bruel \& Kjaer Pulse instrument for measuring vibration data and Pulse Labshop software for analysing frequency response functions. The crack was made by a saw at the $17^{\text {th }}$ element in column \#1. Five levels of the crack from zero to $50 \%$ have been made as can be seen from Fig. 4. These five levels of the crack are presented in Tab. 1.

In this experiment, the frequency response functions were measured by using one hammer and one vibration transducer. The force was applied at a fixed node while the vibration transducer moved along column \#1 as can be seen from Fig. 5. Column \#1 is divided by 36 equal elements. The frequency response functions were obtained at 36 nodes along column \#1. The element stiffness matrix index distribution was then established from these 36 measured frequency response functions by the proposed method.

The experimental results show that there are clear peaks in the element stiffness index distributions of column $\# 1$ at the $17^{\text {th }}$ element with different crack depths as presented in Fig. 6. The significant peak in the element stiffness index distribution explains 


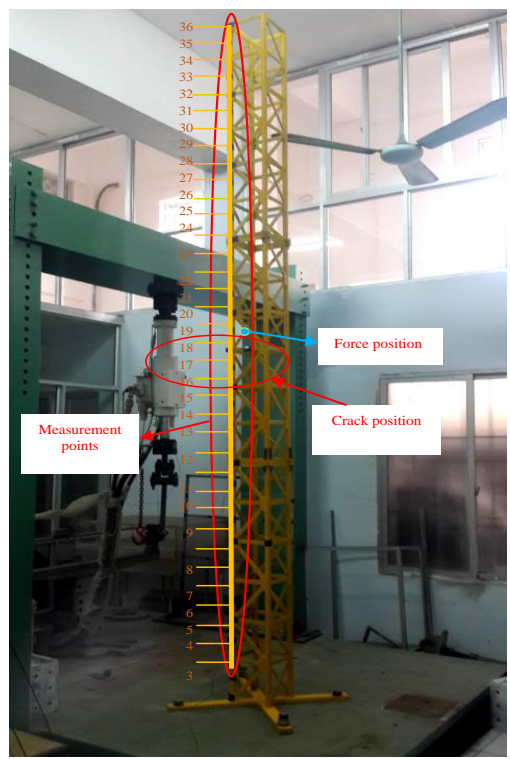

(a)

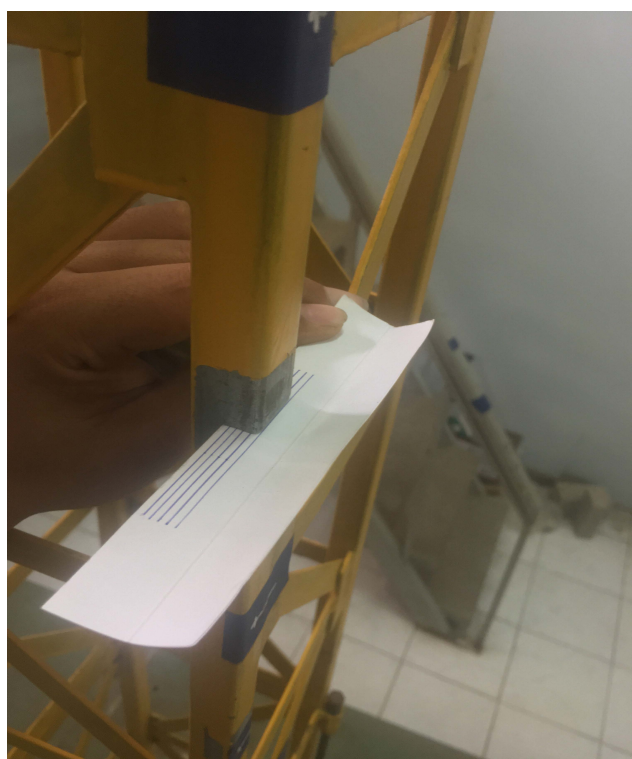

(b)

Fig. 4. Experiment in the laboratory of the Institute of Mechanics - Vietnam Academy of Science and Technology: a) The steel high slender structure; b) Crack was made by a saw

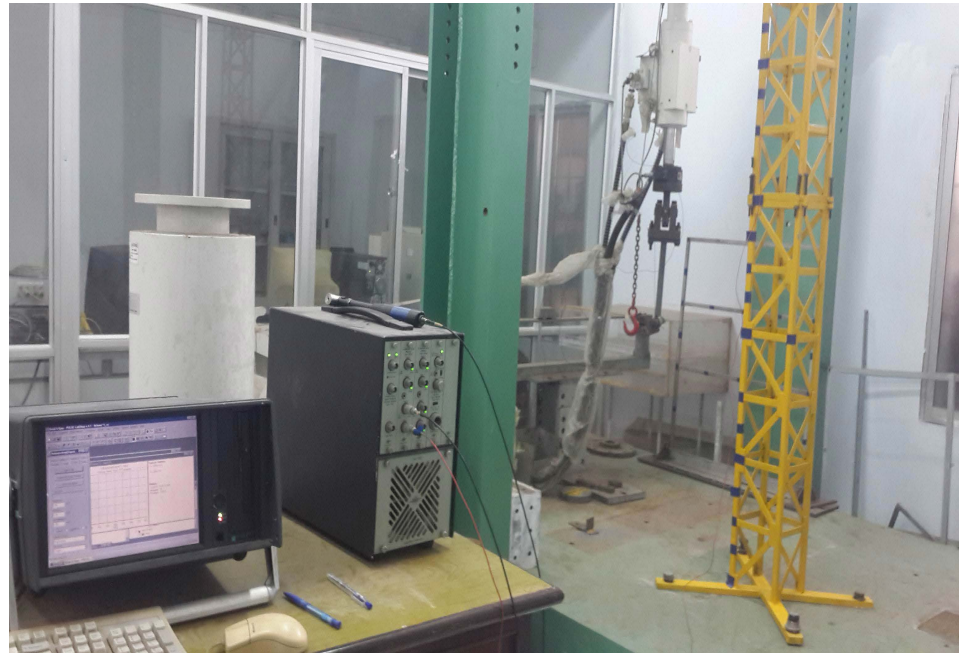

(a)

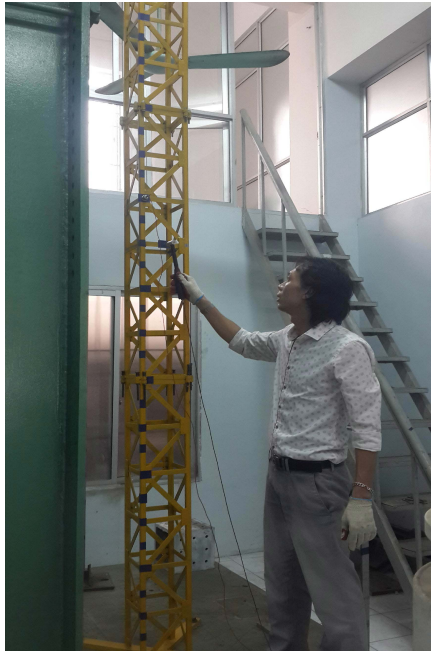

(b)

Fig. 5. Measurement of frequency response functions by PULSE system: a) B\&K Pulse instrument; b) Vibration measurement of the structure acted by hammer 
that there is a change in the stiffness of the $17^{\text {th }}$ element which is the cracked element. Therefore, the peak in the element stiffness index distribution indicates the existence of the crack, and the position of the peak points out the crack location. As can be seen from Fig. 6, when the crack depth increases from $10 \%$ to $50 \%$, the peak at the crack position becomes more significant. This means that when the crack depth is larger the proposed method is more efficient for crack detection.

In order to validate the proposed method for the estimation of crack depth, the relationships between the height of the peak and crack depth in the two cases of simulation and experiment results are presented in the same graph as shown in Fig. 7. It can be observed from Fig. 7 that the relationship between the height of the peak and crack depth in the two cases are similar. Clearly, the simulation and the experimental results are in good agreement to show the applicability of the proposed method in practice.

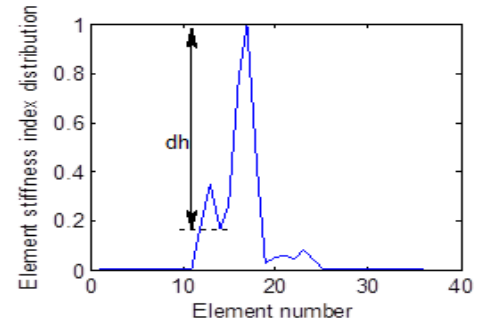

(a)

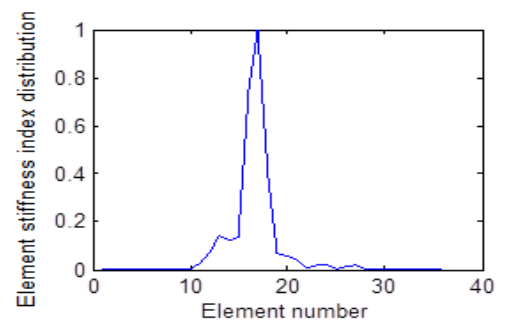

(c)

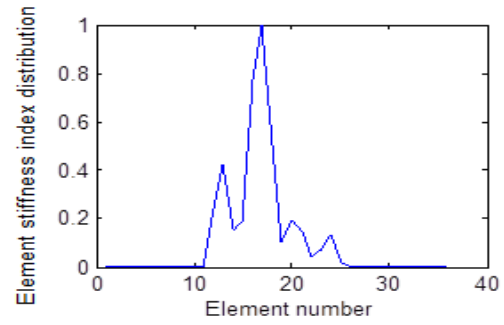

(b)

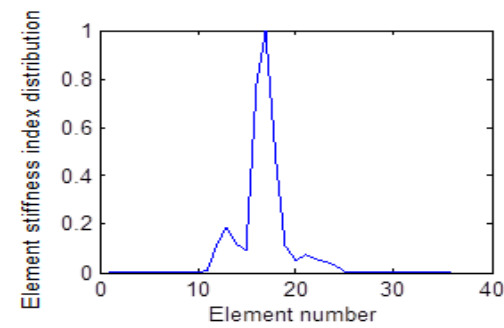

(d)

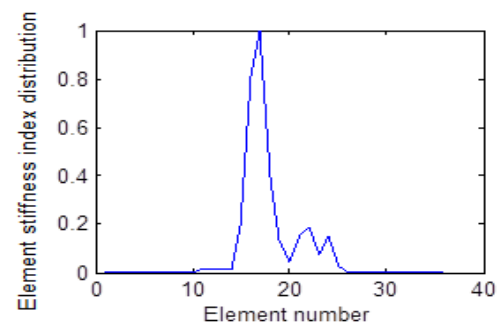

(e)

Fig. 6. Reconstructed element stiffness index distribution, element \#12 is cracked: a) crack depth $10 \%$; b) crack depth $20 \%$; c) crack depth $30 \%$; d) $40 \%$; e) $50 \%$ 


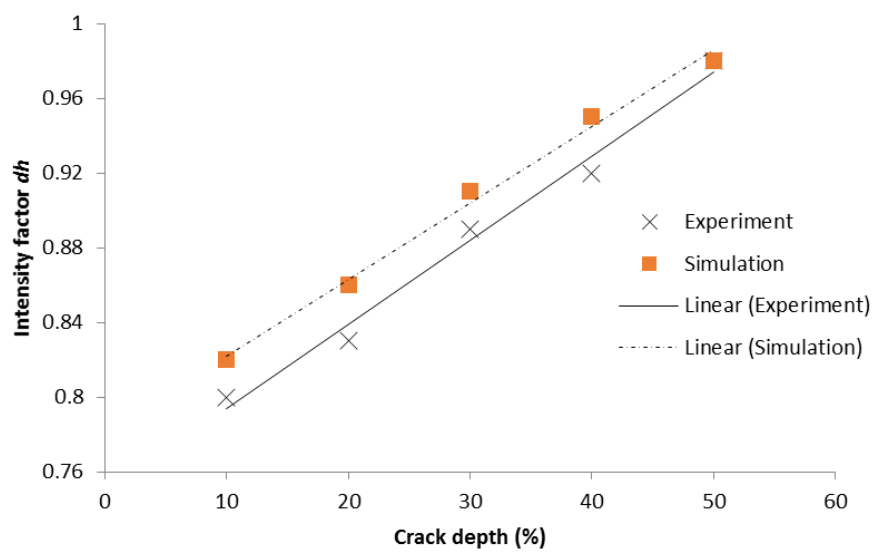

Fig. 7. The height of the first peak versus crack depth

\section{CONCLUSIONS}

In this paper, a method using "element stiffness index distribution" for crack detection of a beam-like structure is presented. The element stiffness index distribution is reconstructed directly from frequency response functions instead of the mode shapes to avoid some limitations of the mode shape-based method for crack detection. Inspecting the change in the element stiffness index distribution, the crack is detected.

The existence of a crack is detected by the significant peak in the element stiffness index distribution. The crack position is determined by the location of the peak. The crack depth can be evaluated from the relationship between the height of peak in the element stiffness index distribution and crack depth. The experiment has been carried out to show that the proposed method can be applied for beam-like structures in practice.

However, the proposed method has its limitation. The accuracy of the detected crack position depends on the number of measurement points since only the cracked element is determined, not the crack position. In order to improve the accuracy of the method more intensive investigation can be made on the crack area. Once the cracked element has been detected, the proposed method can be carried out on the cracked element with more measurement points to determine the more accurate position of crack.

\section{ACKNOWLEDGEMENTS}

This research is funded by Vietnam Academy of Science and Technology under grant number VAST.HTQT.NGA.03/17-18.

\section{REFERENCES}

[1] A. D. Dimarogonas. Vibration of cracked structures: a state of the art review. Engineering Fracture Mechanics, 55, (5), (1996), pp. 831-857. doi:10.1016/0013-7944(94)00175-8.

[2] T. G. Chondros and A. D. Dimarogonas. Identification of cracks in welded joints of complex structures. Journal of Sound and Vibration, 69, (4), (1980), pp. 531-538. doi:10.1016/0022$460 \times(80) 90623-9$. 
[3] R. Y. Liang, J. Hu, and F. Choy. Theoretical study of crack-induced eigenfrequency changes on beam structures. Journal of Engineering Mechanics, 118, (2), (1992), pp. 384-396. doi:10.1061/(asce)0733-9399(1992)118:2(384).

[4] J. Hu and R. Y. Liang. An integrated approach to detection of cracks using vibration characteristics. Journal of the Franklin Institute, 330, (5), (1993), pp. 841-853. doi:10.1016/00160032(93)90080-e.

[5] R. Ruotolo and C. Surace. Damage assessment of multiple cracked beams: numerical results and experimental validation. Journal of Sound and Vibration, 206, (4), (1997), pp. 567-588. doi:10.1006/jsvi.1997.1109.

[6] M.-H. H. Shen and J. E. Taylor. An identification problem for vibrating cracked beams. Journal of Sound and Vibration, 150, (3), (1991), pp. 457-484. doi:10.1016/0022-460x(91)90898-t.

[7] J. Lee. Identification of multiple cracks in a beam using natural frequencies. Journal of Sound and Vibration, 320, (3), (2009), pp. 482-490. doi:10.1016/j.jsv.2008.10.033.

[8] J. A. Loya, L. Rubio, and J. Fernández-Sáez. Natural frequencies for bending vibrations of Timoshenko cracked. Journal of Sound and Vibration, 290, (3), (2006), pp. 640-653. doi:10.1016/j.jsv.2005.04.005.

[9] M. Kisa, J. Brandon, and M. Topcu. Free vibration analysis of cracked beams by a combination of finite elements and component mode synthesis methods. Computers $\mathcal{E}$ Structures, 67, (4), (1998), pp. 215-223. doi:10.1016/s0045-7949(98)00056-х.

[10] A. K. Pandey, M. Biswas, and M. M. Samman. Damage detection from changes in curvature mode shapes. Journal of Sound and Vibration, 145, (2), (1991), pp. 321-332. doi:10.1016/0022460x(91)90595-b.

[11] M. M. A. Wahab and G. De Roeck. Damage detection in bridges using modal curvatures: application to a real damage scenario. Journal of Sound and Vibration, 226, (2), (1999), pp. 217235. doi:10.1006/jsvi.1999.2295.

[12] S. Zhong and S. O. Oyadiji. Detection of cracks in simply-supported beams by continuous wavelet transform of reconstructed modal data. Computers \& Structures, 89, (1), (2011), pp. 127-148. doi:10.1016/j.compstruc.2010.08.008.

[13] A. K. Pandey and M. Biswas. Damage detection in structures using changes in flexibility. Journal of Sound and Vibration, 169, (1), (1994), pp. 3-17. doi:10.1006/jsvi.1994.1002.

[14] B. Jaishi and W.-X. Ren. Damage detection by finite element model updating using modal flexibility residual. Journal of Sound and Vibration, 290, (1), (2006), pp. 369-387. doi:10.1016/j.jsv.2005.04.006.

[15] S. Caddemi and I. Caliò. Exact reconstruction of multiple concentrated damages on beams. Acta Mechanica, 225, (11), (2014), pp. 3137-3156. doi:10.1007/s00707-014-1105-5.

[16] S. Caddemi and I. Caliò. The exact explicit dynamic stiffness matrix of multi-cracked EulerBernoulli beam and applications to damaged frame structures. Journal of Sound and Vibration, 332, (12), (2013), pp. 3049-3063. doi:10.1016/j.jsv.2013.01.003.

[17] K. V. Nguyen and Q. V. Nguyen. Element stiffness index distribution method for multi-crack detection of a beam-like structure. Advances in Structural Engineering, 19, (7), (2016), pp. 10771091. doi:10.1177/1369433216634461.

[18] P. C. Hansen. Regularization tools version 4.0 for Matlab 7.3. SIAM Numerical Algorithms, 46, (2), (2007), pp. 189-194. doi:10.1007/s11075-007-9136-9.

[19] P. C. Hansen. The truncated SVD as a method for regularization. BIT Numerical Mathematics, 27, (4), (1987), pp. 534-553. doi:10.1007/bf01937276.

[20] K. V. Nguyen. Mode shapes analysis of a cracked beam and its application for crack detection. Journal of Sound and Vibration, 333, (3), (2014), pp. 848-872. doi:10.1016/j.jsv.2013.10.006. 